\title{
Random walkers versus random crowds: diffusion of large matrices
}

\author{
Ewa Gudowska-Nowak ${ }^{1}$, Romuald A. Janik ${ }^{1}$, Jerzy Jurkiewicz ${ }^{1}$, \\ Maciej A. Nowak ${ }^{1}$, Waldemar Wieczorek ${ }^{2}$ \\ ${ }^{1}$ M. Smoluchowski Institute of Physics and M. Kac Complex Systems Research \\ Center, Jagiellonian University, Reymonta 4, 30-059 Kraków, Poland \\ ${ }^{2}$ Fachbereich Physik, Universität Duisburg-Essen, 47048 Duisburg, Germany \\ E-mail: gudowska@th.if.uj.edu.pl
}

\begin{abstract}
We briefly review the random matrix theory for large $N$ by $N$ matrices viewed as free random variables in a context of stochastic diffusion. We establish a surprising link between the spectral properties of matrix-valued multiplicative diffusion processes for hermitian and unitary ensembles.
\end{abstract}

PACS numbers: 05.40.+j, 05.45.+b, 05.70.Fh, 11.15.Pg 


\section{Introduction}

Random matrix theory (RMT) represents a powerful tool in several statistical problems where the relevant degrees of freedom can be encoded as elements of certain ensembles of large matrices. Applications cover practically all branches of theoretical physics (spectral properties of excited nuclear, atomic and molecular systems, simplicial gravity, theory of mesoscopic conductance or Euclidean Quantum Chromodynamics) and are more and more frequent in recent interdisciplinary research. Indeed, contemporary complex systems are characterized by massive storage of data, usually cast in the form of matrices. Typical examples are: huge covariance matrices for financial and/or economic indicators, incidence matrices for information/retrieval search engines, adjacency matrices for networks, stochastic matrices describing frequencies of alleles in various gene populations, frequency response matrices in wireless telecommunication systems, to mention only few. Recently, concepts of free random calculus [1, 2] allowed to build in parallel to Classical Probability Calculus(hereafter CPC) a new framework - matrix-valued probability theory, hereafter named Free Probability Calculus (FPC), and allowed to recast several mathematical features of large size random matrices in an intuitive and user-friendly way.

Most of the above-mentioned applications of random matrices of free random variables correspond to the "static case", when we are only interested in spectral properties of certain ensembles, and not in the dynamical properties of evolution of such ensembles as a function of some external parameter, representing either real time or the length of a disordered wire, temperature or the numerical value of the coupling constant. In all these realms matrix ensembles are becoming evolving dynamical systems. This is the problem we are addressing in this note: using the parallel between standard probability calculus for one-dimensional random variables and noncommutative probability calculus of large matrices (FPC) we consider the simplest matrix-valued stochastic evolutions - Brownian walks. Note, that in our case the stochastic increment is represented by a whole matrix, so we deal rather with Brownian crowds than with single walker.

The formulation of this problem was addressed in our previous papers [3, 4, 5], where we have also given references to related literature on this subject. Here, after very brief familiarization with such concepts of free variables as multiplicative S transformation (section 2), we review two simple cases of stochastic multiplicative evolutions considered recently in the literature [3, 5] (unitary multiplicative evolution and hermitian multiplicative evolution) and we establish a new link between their spectral properties. 


\section{S transformation}

One dimensional diffusion process for geometric random walk (in the absence of drift) is given by the stochastic differential equation

$$
\frac{d y}{y}=\sigma d x_{\tau}
$$

where $y$ is random and $d x_{\tau}$ represents Wiener measure. By averaging $y(\tau)$ we recover well known solution for the probability density, so-called log-normal law

$$
p\left(y, \tau \mid y_{0}, 0\right)=\frac{1}{y \sqrt{2 \pi \sigma^{2} \tau}} \exp \left[-\frac{\left(\log \left(y / y_{0}\right)+\frac{1}{2} \sigma^{2} \tau\right)^{2}}{2 \sigma^{2} \tau}\right]
$$

The corresponding analogue in the space of matrices may be written as the product of random matrix-valued variables

$$
Y_{\tau}=\prod_{i}^{K}\left(1+\sqrt{\frac{\tau}{K}} X_{i}\right)
$$

where $X_{i}$ represent large (infinite) hermitian Gaussian free matrices belonging to GUE with the unit variance and infinitesimal time step is equal to $\tau / K$.

To solve this multiplicative diffusion process we notice, that since matrices are non-commuting objects, a standard exponentiation trick relating algebraic to geometric Brownian walk fails. This is why we have to use the concepts of free random variables. We proceed in the following steps.

First, we consider an ensemble built of large $N \times N$ matrices $M$, for which the pertinent resolvent (Green's function) is generically given by

$$
G(z)=\int d M e^{-N V(M)} \frac{1}{N} \operatorname{Tr}\left(\frac{1}{z-M}\right),
$$

In the case of Gaussian ensemble, potential $V(M)$ equals $\frac{1}{2} \operatorname{Tr} M^{2}$. For large $z$, the Green's function is the generating function for spectral moments (we do not consider here so-called Lévy ensembles, for which moments do not exist). In the Gaussian case $G(z)=\left(z-\sqrt{z^{2}-4}\right) / 2$, so the imaginary part yields Wigner's semicircle spectral distribution and expansion in $1 / z$ generates all the spectral moments of this distribution.

Second, note that the product of hermitian matrices is, in general, not a hermitian matrix anymore, which means that the eigenvalues start to diffuse over the whole complex plane, and do not stay on the real axis. This problem was recently solved by us using diagrammatic tools of random matrix theory [3]. Note however, that in the case when we are only interested in moments of the resulting non-hermitian distribution, we may still use the concepts of the generating functions, i.e. resolvents, and considerably reduce the technical complexity of the whole problem. This is due to the fact that even for non-hermitian ensembles, whose eigenvalues lie in bounded domains of the complex plane, the Green's function (4) is analytic around $z=\infty$ and thus serves as a generating function for the moments.

In order to carry out this program in practice, we use here a remarkable tool of FPC known as multiplicative $\mathrm{S}$ transformation, allowing us to find a generating function for all 
moments of the product, provided the spectral properties of the individual ensembles are known. The operational procedure is quite simple: Let us assume that we know Green's $G_{i}(z)$ functions for each of the hermitian ensembles $M_{i}$. We construct an auxiliary analytical function $\chi_{i}$ for each of them, defined by the condition

$$
\frac{1}{\chi_{i}} G_{i}\left(\frac{1}{\chi_{i}}\right)-1=z
$$

and then we redefine the result, getting $S_{i}(z) \equiv \frac{1+z}{z} \chi_{i}(z)$. The resulting S-transform is multiplicative, i.e.

$$
S_{1 \times 2}(z)=S_{1}(z) \cdot S_{2}(z)
$$

and represents the multiplication law for the spectral cumulants.

Applying the concept to individual matrix-valued entry $1+\sqrt{\tau / K} X$, we obviously recover Wigner's semicircle shifted by 1 and with rescaled variance $\beta=\tau / K$. A short calculation [4] yields the desired S-transformation for the product

$$
S_{Y}(z)=\lim _{K \rightarrow \infty} S_{K}=\lim _{K \rightarrow \infty} \prod_{K} S_{i}=\lim _{K \rightarrow \infty}\left(\frac{-1+\sqrt{1+4 \tau z / K}}{2 \tau z / K}\right)^{K}
$$

The surprisingly simple answer reads

$$
S_{Y}(z)=\exp (-\tau z)
$$

Note that despite the fact that we have considered initially only Gaussian ensembles (with unit variance), the above result is valid for any hermitian random matrix ensemble provided its second moment is finite and normalized to 1 . In such a way it represents a new central limit theorem for the product of infinitely many hermitian entries.

Let us now consider a quite different multiplicative evolution, solved recently by two of the present authors [5], namely the spectrum of a unitary operator, represented by an infinite product of unitary, free (independent) increments. Now the evolution operator is represented as

$$
Y_{\tau}=\prod_{i}^{K} \exp \left(i \sqrt{\frac{\tau}{K}} H_{i}\right)
$$

where one assumes only that the second spectral moments of hermitian $H_{i}$ are finite. Note that in this case the spectrum always stays on the unit circle, since the product of unitary matrices is unitary. In this case, the S-transformation is again surprisingly simple and the result reads [5]

$$
S(z)=\exp (\tau(z+1 / 2))
$$

where we have set the arbitrary variance of the initial distribution to one, to simplify the comparison between the unitary and hermitian cases considered here.

\section{Dynamical evolution of both ensembles}

To distinguish between both cases of unitary and hermitian $\mathrm{S}$ transformations, we denote them by $U$ and $H$ subscripts, respectively. Since $S_{H}(z)=\exp (-\tau z)$ and 
$S_{U}(z)=\exp \tau((z+1 / 2)$, we notice that

$$
S_{H}(z) S_{U}(z)=e^{\tau / 2}
$$

i.e. spectral properties of moments are very closely related. To see the relation to moments in more detail, we recall that the Green's function $G(z)$ for the unitary diffusive matrix ensembles [5] generates moments

$$
\frac{1}{z} G\left(\frac{1}{z}\right)-1=\sum_{n=1}^{\infty} m_{n} z^{n}
$$

where

$$
m_{n}(\tau)=<\operatorname{Tr} Y_{\tau}^{n}>=\frac{1}{2 \pi} \int \rho(\theta, \tau) e^{i n \theta} d \theta
$$

Since the multiplicative Voiculescu $\mathrm{S}$ transformation is related to the functional inverse of the generating function:

$$
\frac{1}{\chi(z)} G\left[\frac{1}{\chi(z)}\right]-1=z
$$

where $S(z)=\frac{1+z}{z} \chi(z)$, one can apply standard residua trick for inverting the series [6], getting that

$$
m_{n}=\frac{1}{n} \oint \frac{d z}{2 \pi i}[\chi(z)]^{-n}
$$

In the case of $S_{U}(z)$ the integral representation for moments reads:

$$
m_{n}=\frac{1}{n} \oint \frac{d z}{2 \pi i}(1+1 / z)^{n} \exp (-n \tau(z+1 / 2))=\frac{1}{n} e^{-n \tau / 2} L_{n-1}^{(1)}(\tau n)
$$

where we used the integral representation of the Laguerre polynomials. This result resembles the structure observed in the Gross-Witten type models [7]. A closer look shows, that the similarity to Gross-Witten models or string models is actually exact, provided that the corresponding coupling constant in this model (or the surface of the string in string models) is identified with the evolution parameter $\tau$ (in units where the second spectral moment is one). As far as we now, this simple observation relating the generic models to diffusive properties of large matrices was never explicitly stated in the literature.

\section{Phase transitions}

It is important to notice that both considered models have phase transitions. In the case of unitary diffusions, the phase transition is visible e.g. from the large $n$ behavior of moments. In this case, asymptotics of Laguerre polynomials known as Plancherel-Rotach limit confirms the critical value $\tau_{c}=4$, above which moments decay exponentially and for times smaller than critical, moments oscillate with amplitude $n^{-3 / 2}$. These results were explicitly derived in the context of two-dimensional QCD by Gross and Matytsin [8], provided we identify string surface $A$ with $\tau$. Resulting phase transition is third order (Douglas-Kazakov type), and generic for all types of Gross-Witten type models. It corresponds to a geometrical phase transition, when 
diffusing spectra, originally concentrated at single point or the finite arc of the unit circle, spread in both orientations of the circle to meet and to close the gap previously free of eigenvalues. Our duality relation (11) suggests the presence of phase transition at $\tau_{c}=4$ also for the case of the the product of hermitian ensembles. This was the case observed analytically and confirmed numerically in [3]. The phase transition there was of "topological nature" again - it corresponded to the appearance of the hole on the plane of diffusing complex eigenvalues.

\section{Burgers equations}

Last but not least, we address the issue of dynamical equations describing abovementioned phenomena. To understand the structure of the a-priori unknown equations, we consider first the simple additive evolution of Wigner semicircle. This is the case when the length of support averaged spectral distribution of eigenvalues has to grow as $\sqrt{\tau}$, in analogy to Einstein-Smoluchowski formula for "classical diffusion". This process was already considered in 1962 by Dyson [9], who was considering stochastic differential Langevin equations for temporal behavior of the eigenvalues

$$
d \lambda_{i}(\tau)=\frac{1}{\sqrt{N}} d x_{\tau}^{(i)}+\frac{1}{N} \sum_{i \neq j} \frac{1}{\lambda_{i}(\tau)-\lambda_{j}(\tau)} d t
$$

where the first term represents Wiener process and the second, originating from the measure (van der Monde determinant) represents deterministic, strong repulsive force between any pair of eigenvalues. For large $N$ individual Langevin-like equations may be represented by the equation for average spectral distributions $\rho(\lambda, \tau)$, i.e. analogs of Fick/Fokker-Planck equations known in classical case. They read [10]

$$
\partial_{\tau} \rho(\lambda, \tau)+\partial_{\lambda}\left[\rho(\lambda, \tau) \mathrm{PV} \int \frac{\rho\left(\lambda^{\prime}, \tau\right)}{\lambda-\lambda^{\prime}} d \lambda\right]=0
$$

therefore are non-linear. An alternative way to recast these equations is to use the Green's function. Since imaginary part of the Green's functions is related to spectral density, matrix-valued "diffusion equations" take the form

$$
\partial_{\tau} G(z, \tau)+G(z, \tau) \partial_{z} G(z, \tau)=0
$$

known also as complex Burgers equation.

We can return now to the case of the hermitian multiplicative diffusion. Knowing $S_{H}(z)=\exp (-\tau z)$ we read out the Green's function for the ensemble $Y$

$$
G_{Y}(z, \tau)=\frac{1+f(z, \tau)}{z}
$$

where $f$ is the solution of the transcendental equation

$$
z f=(1+f) e^{\tau f}
$$


Note, that by definition, $G_{Y}(z, \tau)$ is a holomorphic function (defined for large $z$ ) generating spectral moments of $Y_{\tau}$, i.e.

$$
G_{Y}(z, \tau)=\sum_{n=0}^{\infty} \operatorname{tr} Y_{\tau}^{n} \frac{1}{z^{n+1}}
$$

Let us remind [4] that $S_{Y}$ fulfills a simple equation

$$
z \partial_{z} S-\tau \partial_{\tau} S=0
$$

By differentiating (21) with respect to $\tau$ and $z$ we get the following evolution equation

$$
\partial_{\tau} f+z f \partial_{z} f=0
$$

or equivalently

$$
\partial_{\tau} f+f \partial_{\ln z} f=0
$$

supplemented by the boundary condition $f(\tau=0, z)=(z-1)^{-1}$. This is the Burgers equation where spatial evolution is governed by $\log z$. Note however, that this time the semicircle solution of the additive Burgers equation (19), with $z$ replaced by $\ln z$, does not fulfill (24). It should not be puzzling for the reader that similar Burgers-like equations describing the evolution of the spectra one encounters also in the case of unitary evolutions [5, 8].

\section{Summary}

In this paper we discussed some problems of Brownian-like walks in the space of the spectra of large matrices with the help of powerful tools of free random variables calculus. New results presented in this note include: (i) a rather unexpected link between the moments of unitary evolutions and hermitian evolutions; (ii) an exact correspondence between diffusive character of evolving large unitary matrices and the whole class of generic models of Gross-Witten type known in two-dimensional Quantum Chromodynamics. We hope that the language of matrix-valued evolution presented here does not only form an exciting and rich mathematical structure, but may also help to understand basic concepts of "new statistical physics" of large and non-commuting objects.

Acknowledgments This work was supported in part by the Polish State Committee for Scientific Research (KBN) grants 2P03B08225 (2003-2006), 1P03B 02427 (20042007) and 1P03B04029 (2005-2008) and the Marie Curie Actions Transfer of Knowledge project COCOS (contract MTKD-CT-2004-517186). Waldemar Wieczorek acknowledges a financial support within the DFG programme SFB/TR12. The authors

are grateful to Roland Speicher and Piotr Śniady for valuable remarks on Free Random Variables calculus, and to Poul Olesen for the correspondence on the link with 2D YM. 


\section{References}

[1] Voiculescu D.V. 1991 Inv. Math. 104201.

Voiculescu D.V., Dykema K. and Nica A. 1992 Free Random Variables (Providence, RI: CRM Monograph Series Vol.1, Amer. Math. Soc.)

[2] Speicher R. 1992 Math. Z. 20955

Bozejko M., Leinert M., Speicher R. 1996 Pac. J. Math. 175357.

[3] Gudowska-Nowak E., Janik R.A., Jurkiewicz J. and Nowak M.A. 2003 Nucl. Phys. B670 479

[4] Gudowska-Nowak E., Janik R.A., Jurkiewicz J. and Nowak M.A. 2005 New Jour. Phys. 754

[5] Janik R.A. and Wieczorek W. 2004 J. Phys. A 376521

[6] Morse P.M. and Feshbach H. 1953 Methods of Theretical Physics vol.1 (Mc Graw-Hill, New York), page 411.

[7] Gross D. and Witten E. 1980 Phys. Rev D21 44

[8] Gross D. and Matytsin A. 1995 Nucl. Phys. B437 541 and references therein.

[9] Dyson F.J. 1962 J. Math, Phys. 31191

[10] Biane Ph. and Speicher R. 2001 Ann. Inst. Henri Poincaré PR 37581. 